\title{
Prospects of Resonant Optical Antennas for Nano-Analysis
}

\author{
Bert Hecht ${ }^{\star a}$, Peter Mühlschlegel ${ }^{\mathrm{a}}$, Javad N. Farahani ${ }^{\mathrm{a}}$, Hans-Jürgen Eisler ${ }^{\mathrm{a}}$, Dieter W. Pohla \\ Olivier J.F. Martin ${ }^{\mathrm{b}}$, and Paolo Biagionic
}

\begin{abstract}
Suitably shaped metal nanostructures act as resonant optical antennas that efficiently collect light and confine it to a subwavelength volume. Vice versa, light emission from nano volumes can be enhanced by coupling to antenna structures. We give a short introduction to antenna theory and discuss recent experiments that show the feasibility of achieving strong field enhancement using resonant dipole antennas for near infrared wavelengths. By scanning an optical antenna fabricated at the apex of an AFM tip over individual quantum dots, we observe enhanced emission of the latter while it is in close proximity of the antenna feed gap. Resonant optical antennas hold promise to be applied for spectroscopic characterization of nano structures with high spatial resolutions and single-molecule sensitivity.
\end{abstract}

Keywords: Atomic force microscope (AFM) · Metal nano structures · Resonant optical antennas

\section{Introduction}

Optical spectroscopy provides a unique way to learn about the chemical nature of unknown materials [1]. In combination with optical microscopy techniques that provide two- or three-dimensional spatial resolution, powerful methods of chemical imaging are created. Optical techniques are fully compatible with investigations at ambient conditions and in liquids, which explains their ever-increasing importance for life science applications. Particularly useful

\footnotetext{
${ }^{*}$ Correspondence: Dr. B. Hecht ${ }^{\mathrm{a}}$

E-Mail: bert.hecht@nano-optics.ch

aNano-Optics group

National Center of Competence for Research in $\mathrm{Na}$ -

noscale Science

Institute of Physics

University of Base

Klingelbergstr. 82

$\mathrm{CH}-4056$ Basel

bNanophotonics and Metrology Laboratory

Swiss Federal Institute of Technology Lausanne (EPFL)

ELG 240, Station 11

$\mathrm{CH}-1015$ Lausanne

cDipartimento di Fisica

Politecnico di Milano

Piazza L. da Vinci 32

20133 Milano, Italy
}

types of optical spectroscopy, for example Raman and infrared spectroscopy [2], are related to the identification of the molecules under investigation since the vibrational modes involved are uniquely dependent on the molecular structure. Another vast field of important and successful applications of optical microscopy is the ultrasensitive and highly specific detection of fluorescent labels predominantly in biological environments [3][4]. Here a structure of interest is labeled with a fluorescent dye or another type of chromophore whose fluorescence is detected in a spectral window free of other emission. Single dye-molecule sensitivity is easily achievable in such experiments which reduces the necessary invasiveness of the labeling process and opens possibilities to trace molecular interactions in real time [5].

An obvious goal for the future development of such optical techniques for nanoanalyses is to increase their spatial resolution into the sub-10 nm regime. Recently enormous progress has been made to increase the resolution of far-field microscopy into the sub-20 $\mathrm{nm}$ regime, using specific dye molecules for staining together with an illumination scheme involving two pulsed lasers for point-spread function shaping [6]. However, spectroscopic experiments with high spatial resolution require true field confinement on a length scale similar to the desired spatial resolution. Such confined optical fields are created by the illumination of, preferably metallic, material structures [7]. The external field induces oscillations of the material electrons which re-radiate a secondary field. The near-field of this secondary field is a quasi-static field which oscillates at the optical frequency. The relevant near-field distribution can in this approximation be inferred from electrostatics and is for numerous geometries intuitively clear or documented in the literature [8]. A beneficial side effect of the field confinement is the concomitant field enhancement with respect to the driving field. It is due to evanescent modes that contribute to the fields in close proximity to the material structure. Using field enhancing and confining optical probes scanning near-field optical imaging and spectroscopy of samples can be performed with a spatial resolution determined solely by the degree of field confinement.

Examples for relevant probe structures are subwavelength metallic spheres and sharp metal tips. In both cases the small radius of curvature hints at the presence of localized and enhanced optical fields. The field confinement that is achievable using such structures has recently been demonstrated experimentally for both geometries. The expected field confinement and enhancement has been verified. Sharp noble metal tips were used to study the Raman scattering from carbon nanotubes at resolutions approaching the $10 \mathrm{~nm}$ barrier [9]. Small gold spheres were brought in close proximity to single dye molecules that showed enhanced fluorescence when the gold sphere was scanned in a large enough distance above the molecule [10][11]. For 
very small distances complete quenching of the fluorescence was observed. The finite distance that has to be kept limits the achievable resolution since the confinement strongly decreases with distance. Even very small gold particles are found to completely quench nearby fluorescent molecules [12] which implies that very small metal structures might not be a solution to this problem.

Here we review progress made towards the application of optimized metal structures, called 'resonant optical antennas', as novel field-confining optical probes for scanning near-field optical microscopy and spectroscopy [13-15]. Resonant optical antennas are downscaled replicas of their radio wave counterparts [16][17]. However, it is important to note the finite conductivity of metals at optical wavelengths as well as the existence of surface plasmon resonances [18] which lead to considerable deviations from classical antenna textbook knowledge. The advantage of resonant optical antennas with respect to less favorable probe designs is the fact that resonant optical antennas provide optimal conversion of localized fields into propagating fields. It may thus be hoped that it will be possible to increase the contribution of radiative decay channels of single emitters by placing them close to the nanometer-sized feed gap of a resonant optical antenna. In addition, the antenna may redistribute the emitted radiation in a favorable way. Therefore, both fluorescence and Raman experiments will benefit from using resonant optical antennas as probes.

We start out by giving a short intuitive introduction into field confinement and enhancement in the antenna feed gap as a function of antenna length for thin dipole antennas. We then review experimental results that demonstrate antenna-related field enhancement at near infrared wavelengths. Finally, we discuss first experiments to utilize antenna probes for scanning near-field optical microscopy.

\section{Field Confinement and Enhancement in Thin Dipole Antennas}

In classical antenna theory an antenna structure is described completely when the distribution of currents on the antenna is known. Once the current distribution as the source of the radiation field is known, the emitted radiation can be easily calculated using Green's function formalism. This is true for the receiving antenna which is subjected to an external radiation field as well as for an emitting antenna which is driven by a near-field source in the feed gap. For a thin linear dipole antenna there is a particularly simple way to obtain a first approxi- mation for the one-dimensional current distribution. Consider an open two-wire waveguide which is fed by some high-frequency generator (see Fig. 1). Due to the open end most of the energy traveling down the waveguide is reflected and a standing wave builds up. This configuration is a very bad antenna since hardly any power is emitted into the far field although strong nearfields fields exist between the two wires. This can be understood considering the currents in the waveguide. Each current element in one wire has its counterpart in the other wire albeit with an $180^{\circ}$ phase shift. Since the distance between the two wires is much smaller than the characteristic wavelength, the radiation of each pair of current elements cancels in the far field.

The situation changes dramatically if the parts of the wire with a well-defined length are bent up and down as sketched in Fig. 1(b). Now the current elements in the wire oscillate in phase and electromagnetic waves can effectively be emitted. Fig. 1(c) shows current distribution and the corresponding charge distribution that can be obtained via the continuity equation for charges applied to a one-dimensional current. For a total length $L$ of the antenna of $\lambda / 2$ where $\lambda$ is the wavelength, the charge accumulation near the antenna feed gap is largest leading to very strongly enhanced fields in this region. For longer antennas, e.g. $L=\lambda$, although the potential current amplitude is larger this mode cannot be excited by an excitation in the gap since the current amplitude there is zero. The other way round such an antenna cannot be used to receive e.m. waves through enhanced fields over the gap.

\section{Fabrication of Optical Dipole Antennas}

To achieve antenna-like effects at optical frequencies we constructed gold nano dipole antennas with overall lengths ranging from 200 to $400 \mathrm{~nm}$. To fabricate such tiny structures in a $40 \mathrm{~nm}$ gold film on a thin ITO-coated cover glass we apply a two-step process. It consists of an initial step which defines raw structures by electron beam lithography and a fine-processing step dur-

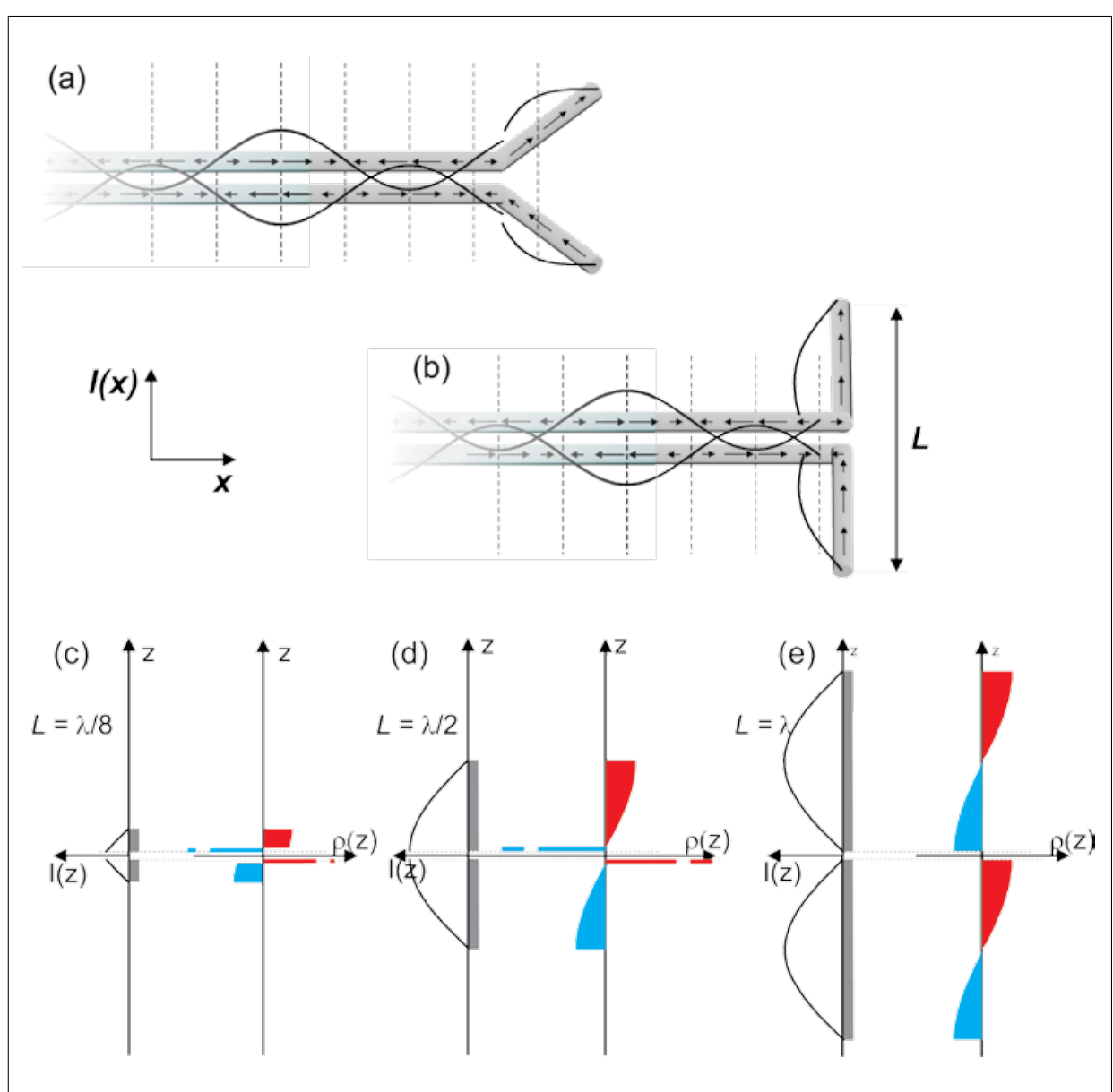

Fig. 1. Antenna theory in a nutshell. (a), (b): Two-wire waveguide with an open end sustaining a standing wave. By bending up the ends an efficient antenna is created with overall length I. The unmodified waveguide is a bad emitter since the out-of-phase current elements in close proximity do not contribute to a far-field radiation field. (c), (d), (e): Current distribution on dipole antennas of increasing length as indicated. The respective charge distributions are also sketched (red: pos. charge, blue: neg. charge). The maximum charge accumulation and therefore the highest field in the feed gap is obtained for $L=\lambda / 2$. 
ing which the antenna structures are milled from the raw structure. Fig. 2 outlines the sequence of fabrication steps. Figs 2(a)-(c) show successive SEM zooms into a field of gold metal patches on glass. Relatively large finding structures (triangles) are necessary to find the patch array in the optical microscope. The distance between patches is large to ensure that they are not coupled. Fig. 2(d) shows the milling pattern that was applied and its sequence. Five rectangular areas of the patch are removed sequentially leaving behind a well-defined dipole nanoantenna consisting of two gold rods separated by a narrow gap: the feed gap. Using the same process dipole antennas of various lengths have been fabricated [14].

Gold is known to exhibit two-photon excited photoluminescence [19]. To see if these structures exhibit field enhancement they were subjected individually by means of scanning confocal optical microscopy to a pulsed infrared laser with a pulse duration of $8 \mathrm{ps}$ at a wavelength of $830 \mathrm{~nm}$. The average power at a repetition rate of $80 \mathrm{Mhz}$ was below $100 \mu \mathrm{W}$. The stronger the field enhancement on the structure the stronger the luminescence effect is expected to be. Surprisingly, for resonant antennas in addition to the expected luminescence we also observe very strong light emission with a fourth-order power dependence which we assign to white-light continuum generation - most probably in the antenna feedgap [14]. The observed fourth-order power dependence is a strong indication for the presence of strong field enhancement. Fig. 3 shows the result of such experiments as a function of the antenna length. Fig. 3(a) shows zooms of a too long, a resonant and a too short antenna. In Fig. 3(b) overview images are presented showing the orientation of the antenna which can be compared to scanning confocal optical images; Figs 3(c) and (d) are recorded at the same position. It is easy to see that the white-light continuum signal is largest for the resonant antenna for a polarization of the excitation beam along the antenna axis. Fig. 3(e) shows a plot of the maximum white-light emission $v s$. the antenna length in a semi-logarithmic plot (squares). The curve is a result of a field simulation based on the Green's tensor technique [20]. Remarkably the calculated maximum of the field enhancement coincides very well with that found experimentally [14].

\section{Bowtie Antenna AFM Probes}

So far our results show that it is possible to create a receiving antenna structure for light. For the field of optical nano analysis, as we pointed out in the introduction, it would be very helpful if similar antenna structures could be used
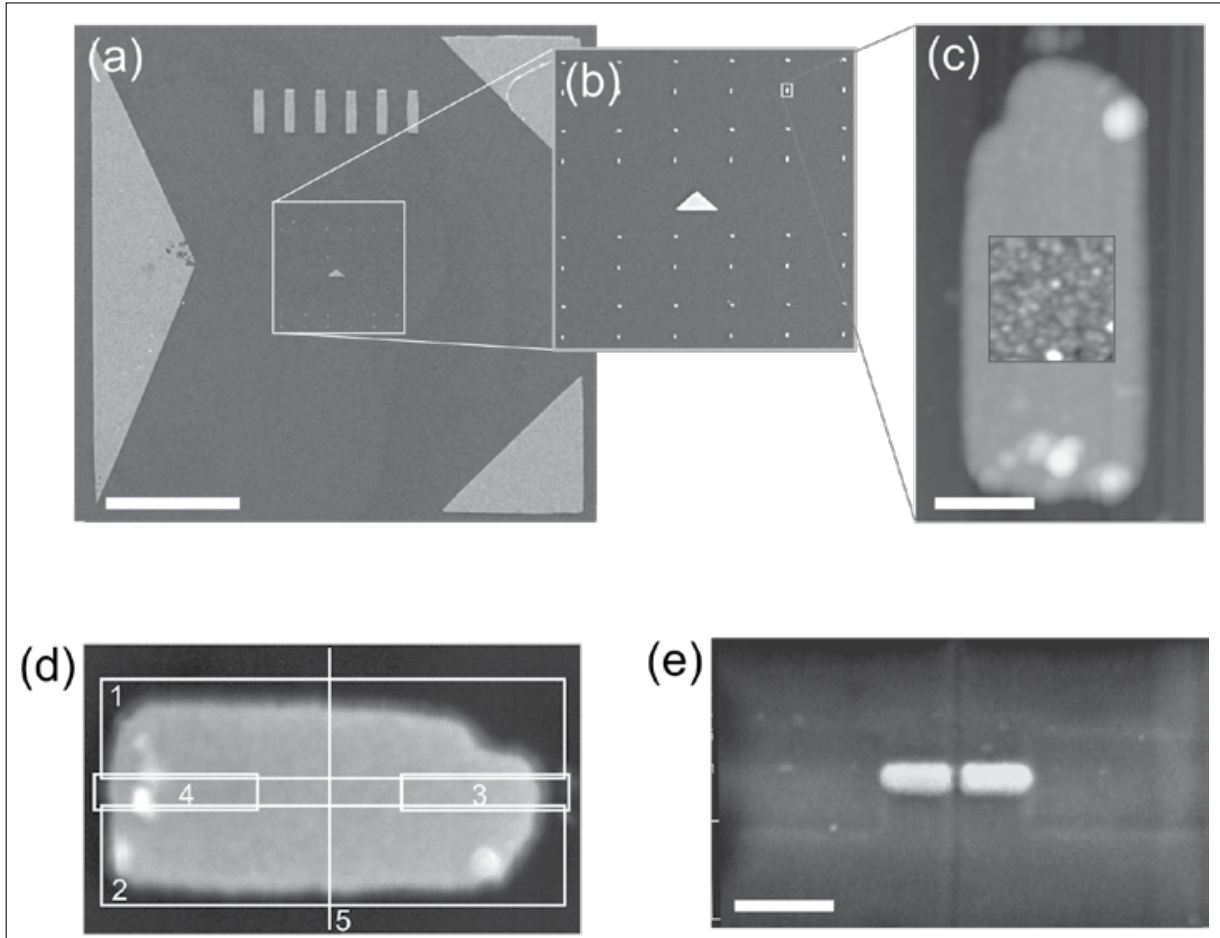

Fig. 2. Fabrication steps. (a): SEM overview after metal patch fabrication using electron beam lithography. Scale bar $50 \mu \mathrm{m}$. (b): Zoom $\left(50 \times 50 \mu \mathrm{m}^{2}\right)$ on the array of metal patches. (c): AFM-image of one gold patch $\left(400 \times 800 \times 40 \mathrm{~nm}^{3}\right)$ before focused-ion beam milling. The inset shows a high resolution AFM-image $\left(250 \times 250 \mathrm{~nm}^{2}\right)$ used for surface roughness determination. (d): Milling pattern sequence (numbers) applied during focused-ion beam milling of the structure in (a). (e): Resulting optical antenna. Scale bar $200 \mathrm{~nm}$.

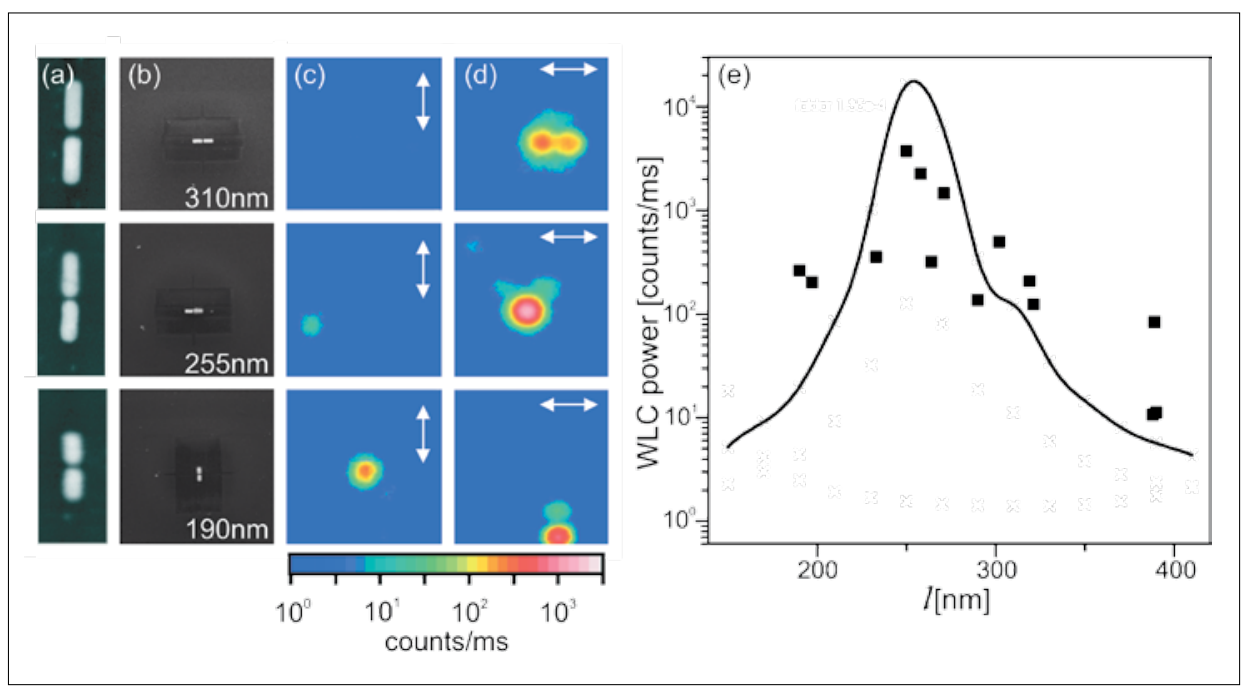

Fig. 3. Antenna characterization. (a), (b): SEM close-up $\left(450 \times 180 \mathrm{~nm}^{2}\right)$ and overview $\left(2 \times 2 \mu \mathrm{m}^{2}\right)$ showing the detailed shape as well as the orientation and the immediate surrounding of the antenna structures. (c), (d): Scanning confocal optical images for perpendicular polarizations as indicated by the arrows obtained with pulsed excitation at $\lambda=830 \mathrm{~nm}$ as described in the text. Strong emission is observed only for correct polarization along the antenna structure and for antennas with the correct length. Note the logarithmic intensity scale. (e): Peak emission rate vs. antenna length. The squares are experimental data, the line results from a simulation of the near field $10 \mathrm{~nm}$ above the structure. Remarkably, the observed maximum emission antenna length perfectly coincides with the prediction.

as emitting antennas. The reciprocity theorem for antennas states that a good receiving antenna is also a good emitting antenna if driven by a suitable local field [16][17]. For this purpose the analyte has to be brought into close proximity of the antenna feed gap. One way to achieve this is to fabricate an antenna structure at the apex of a scanning probe, as sketched in Fig. 4(a). The latter highly efficient optical probe may then be raster-scanned over a surface of interest. 
Such resonant antenna probes will

(i) increase the coupling of light into the far-field;

(ii) provide an enhanced excitation intensity, and

(iii) might redirect the emission of photons to favorable directions.

Figs. 4(b) and (c) show the top view as well as the side view, both with a zoomed version in the inset, of an antenna probe fabricated on top of a gold-coated $\mathrm{Si}_{3} \mathrm{~N}_{4}$ AFM cantilever tip using focused-ion beam milling. The bowtie shape of the antenna is adapted to the pyramidal geometry of the AFM tip. The overall antenna length is compatible with the wavelength of visible light. The gap size is rather large $(\approx 50 \mathrm{~nm})$ since the milling at the tip went deep into the $\mathrm{Si}_{3} \mathrm{~N}_{4}$ substrate material creating two $\mathrm{Si}_{3} \mathrm{~N}_{4}$ pillars with a gold cap.

Experiments were performed using antenna probes to demonstrate the possibility of using an optical antenna structure to enhance the emission from a nano volume. To this end single semiconductor nanocrystals (quantum dots) were spincoated onto a glass cover slip and then overcoated with a thin film of PMMA. The film is so thin that the quantum dots still stick out of the film as sketched in Fig. 4(a). Now scanning confocal optical microscopy is used to locate individual nano crystals on the surface. Once a quantum dot is found it is kept fixed in the focus and illuminated continuously at a very low intensity level using a pulse laser $(\lambda=532 \mathrm{~nm}$, rep. rate $=80 \mathrm{MHz}$, pulse length $=10 \mathrm{ps}$ ). Now the antenna probe is approached to the surface and scanned in contact mode through the focus where it eventually will come across the quantum dot. The experiment is sketched in Fig. 4(a). To fully characterize the photon emission both the excited state lifetime and the emission rate of the quantum dot are recorded as a function of the tip position. The results of such an experiment are summarized in Fig. 5. Fig. 5(a) shows the map of the emission rate scanned from top to bottom. As long as the tip is far away, the emission rate of the quantum dot is constant, only interrupted by the typical blinking events that occur on all timescales. As the antenna probe is approached to the quantum dot eventually light reflected at the tip starts to slightly modulate the excitation. However, once the antenna probe is above the quantum dot its emission is clearly enhanced. This is a remarkable finding since typically one would expect that luminescence is quenched in close proximity to a metal [21]. The size of the field enhanced area is compatible with the feed gap width of the antenna probe visible in Fig. 4(c). As an illustration representative decay curves are displayed using all photons collected within a $3 \times 3$ pixel region marked by the white and black squares in Fig. 5(a). While the unperturbed quan-
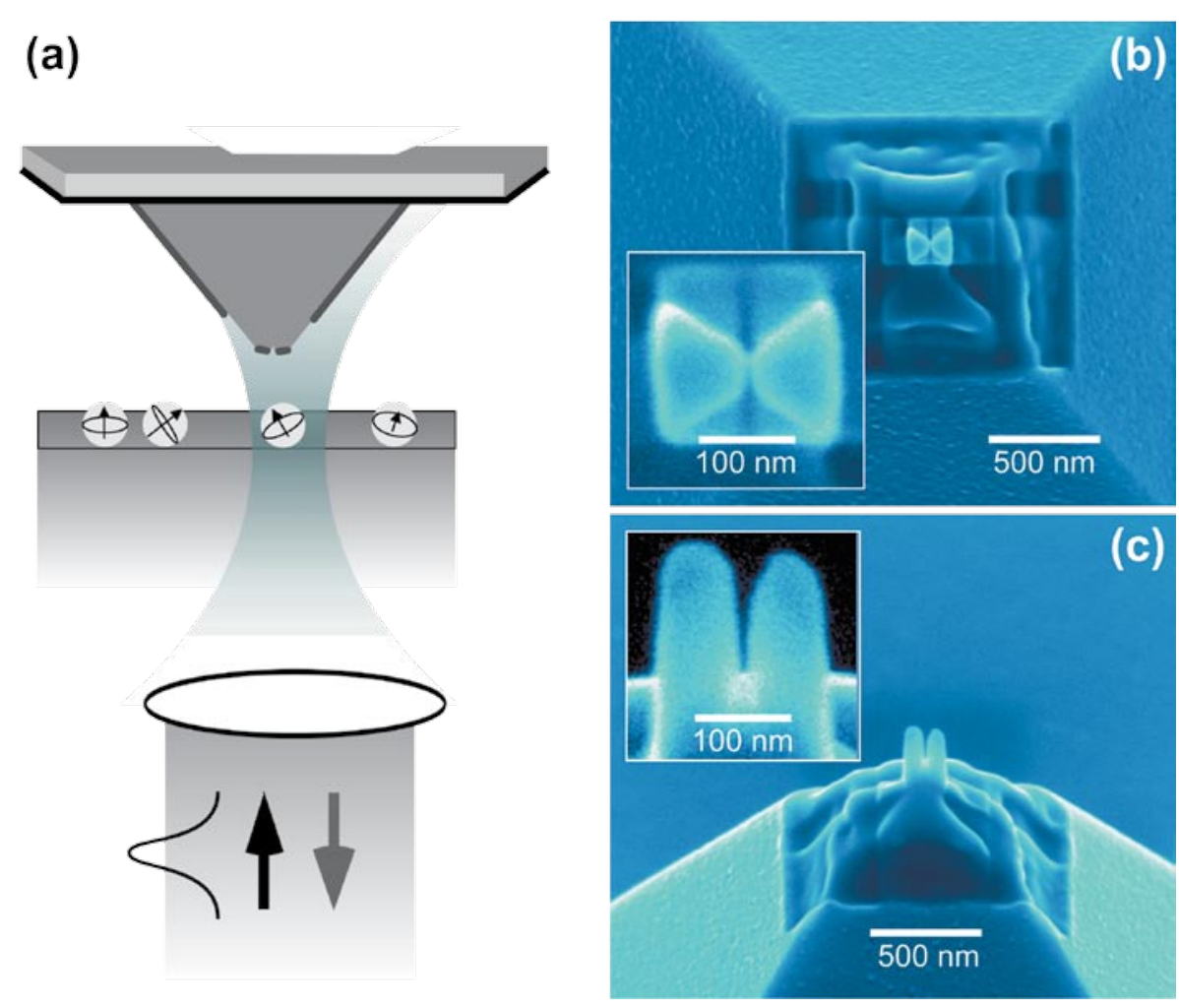

Fig. 4. Towards antenna-based local spectroscopy. (a): Sketch of the experimental configuration (b), (c): Antenna AFM probe fabricated by focused-ion beam milling. Scanning electron microscopy images; top view and side view, respectively.

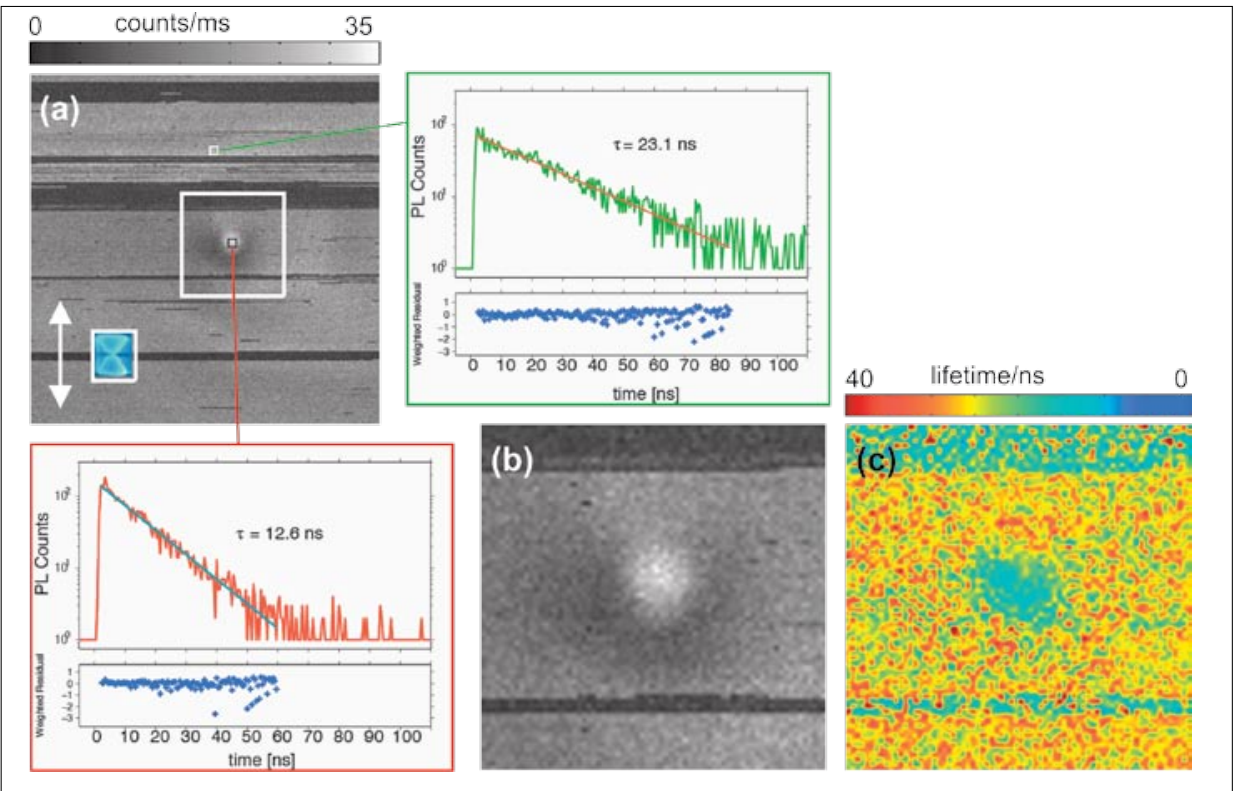

Fig. 5. Antenna enhanced emission of single emitters. (a): Emission rate of a single quantum dot as a function of the antenna probe position. A clear enhancement of the emission rate is observed when the quantum dot couples to the antenna. Inset: Antenna size (to scale). The arrow denotes the direction of polarization used for illumination. At the position of the white and the black square excited-state decay curves are displayed showing a strong reduction of the excited-state lifetime when the quantum dot couples to the antenna. (b), (c): Zoom to the area of the large white square in (a) for both the emission rate and the excited-state lifetime.

tum dot has a lifetime of $23 \mathrm{~ns}$, the lifetime of the quantum dot in contact with the antenna is reduced to $12.6 \mathrm{~ns}$. In combination with the enhanced emission it is plausible to assume that the antenna enhances the radiative decay rate of the quantum dot. Figs 5(b) and (c) show zooms of the emission rate map and the simultaneously recorded lifetime map, respectively [15].

The fact that emission enhancement is observed in the just described experiment is promising, however, the amount of en- 
hancement is rather limited. In view of the strong field enhancements expected from the white-light generation experiment described above, one would expect a much larger enhancement factor. One reason for this finding lies in the difficulty to prepare an antenna structure at the very apex of the probe. Slight misalignments result in asymmetries that result in degraded performance. The same is true for too large feed gaps that result from unreliable determination of the milling dose at the tip apex. To improve upon this problem we have started to prepare AFM tips with a flat end facet at the tip (see Fig. $6)$. On top of this facet well-defined antennas may be fabricated. We expect much higher enhancement factors to be observable from such second-generation optimized probes. Such a development will be instrumental for the use of optical antenna probes for nano imaging and spectroscopy.

\section{Summary}

We have introduced the concept of optical antennas as a promising tool on the way to routine spectroscopic characterization of materials on the nanometer scale. Using the example of a simple dipole antenna we have shown that a length-dependent variation of the field enhancement in the feed gap occurs that has its first maximum at a length of about half the wavelength. We further discussed the experimental implementation of optical antenna structures and the observation of white-light continuum generation for resonant structures. Having demonstrated the antenna concept we have described our first steps towards the development of antenna-based scanning near-field optical microscopy that should provide spatial resolution given by the width of the feed gap $(\approx 10 \mathrm{~nm})$ and a sensitivity to detect even single fluorescent molecules.

\section{Acknowledgements}

The authors acknowledge H.-J. Güntherodt for continuous support. Financial support by the Swiss National Science Foundation is acknowledged via the National Center of Competence in Research (NCCR) in Nanoscale Science and a research professorship for one of the authors (B.H.).

Received: September 21, 2006
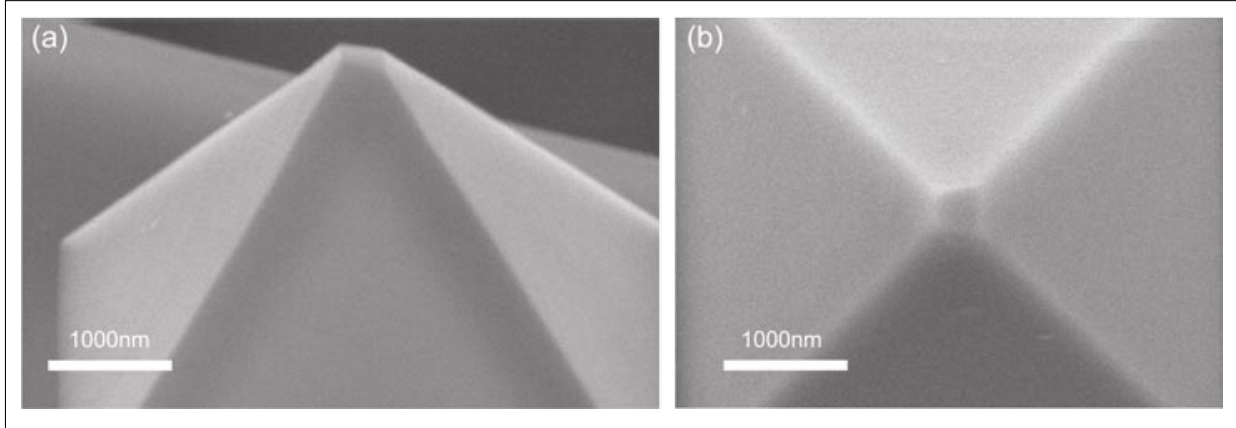

Fig. 6. Towards the next generation of probes. (a): Side view and (b) top view of $\mathrm{Si}_{3} \mathrm{~N}_{4}$ AFM tips (scanning electron microscopy images) that have been flattened by laser machining. The flat end face will allow the preparation of much better defined antenna structures in the future.

[1] 'Frontiers of Optical Spectroscopy', Eds. B. Di Bartolo, O. Forte, Vol. 168 of NATO Science series, Kluwer Academic Publishers, Amsterdam, ii. mathematics, physics and chemistry edition, 2005.

[2] 'Infrared and Raman Spectroscopy. Methods and Applications', Ed. B. Schrader, Wiley VCH, Weinheim, 1998.

[3] 'Single-Molecule Optical Detection, Imaging and Spectroscopy', Eds. T. Basché, W.E. Moerner, M. Orrit, U.P. Wild, VCH Verlagsgesellschaft, Weinheim, 1997.

[4] 'Single-Molecule Detection in Solution', Eds. R.A. Keller, C. Zander, J. Enderlein, Wiley-VCH Verlag GmbH, Weinheim, 2002.

[5] T. Schmidt, G.J. Schütz, W. Baumgartner, H.J. Gruber, H. Schindler, Proc. Natl. Acad. Sci. USA 1996, 93, 2926-2929.

[6] T.A. Klar, S. Jakobs, M. Dyba, A. Egner, S.W. Hell, Proc. Natl. Acad. Sci. USA 2000, 97, 8206-8210.

[7] L. Novotny, B. Hecht. Principles of Nano-Optics. Cambridge University Press, Cambridge, 2006

[8] J.B. Jackson, S.L. Westcott, L.R. Hirsch, J.L. West, N.J. Halas, Appl. Phys. Lett. 2003, 82, 257-259.

[9] A. Hartschuh, M.R. Beversluis, A. Bouhelier, L. Novotny, Phil. Trans. R. Soc. Lond. A 2004, 362, 807-819.

[10] P. Anger, P. Bharadwaj, L. Novotny, Phys. Rev. Lett. 2006, 96, 113002.

[11] S. Kühn, U. Hakanson, L. Rogobete, V. Sandoghdar, Phys. Rev. Lett. 2006, 97, 017402.

[12] E. Dulkeith, A.C. Morteani, T. Niedereichholz, T.A. Klar, J. Feldmann, S.A. Levi, F.C.J.M. van Veggel, D. N. Reinhoudt, M. Möller, D.I. Gittins, Phys. Rev. Lett. 2002, 89, 203002.
[13] P.J. Schuck, D.P. Fromm, A. Sundaramurthy, G.S. Kino, W.E. Moerner, Phys. Rev. Lett. 2005, 94, 017402.

[14] P. Mühlschlegel, H.-J. Eisler, O.J.F. Martin, B. Hecht, D.W. Pohl, Science 2005, 308, 1607-1609.

[15] J.N. Farahani, D.W. Pohl, H.-J. Eisler, B. Hecht, Phys. Rev. Lett. 2005, 95, 017402.

[16] C.A. Balanis, 'Antenna Theory: Analysis and Design', John Wiley \& Sons, Inc., New York, Chichester, 2nd ed., 1997.

[17] K.F. Lee, 'Principles of Antenna Theory', John Wiley \& Sons, Inc., The Chinese University of Hong Kong, 1st ed., 1984.

[18] D.W. Pohl, 'Near field optics seen as an antenna problem', in 'Near-Field Optics: Principles and Applications', Eds M. Ohtsu, X. Zhu, Singapore, 2000, World Scientific, ISBN 981-02-4365-0, p. 9-21.

[19] M.R. Beversluis, A. Bouhelier, L. Novotny, Phys. Rev. B 2003, 68, 115433-10.

[20] M. Paulus, O.J.F. Martin, J. Opt. Soc. Am. A 2001, 18, 854-861.

[21] W. Trabesinger, A. Kramer, M. Kreiter, B. Hecht, U.P. Wild, Appl. Phys. Lett. 2002, 81, 2118-2120. 